\title{
Observations of PSR J2021+3651 and its X-ray Pulsar Wind Nebula G75.2+0.1
}

\author{
J. W. T. Hessels, M. S. E. Roberts ${ }^{1,2}$, S. M. Ransom ${ }^{1}$, V. M. Kaspi, \\ Department of Physics, McGill University, Montreal, H3A-2T8, Canada; \\ hessels@physics.mcgill.ca \\ R. W. Romani, C.-Y. Ng, \\ Department of Physics, Stanford University, Stanford, CA 94305 \\ P. C. C. Freire \\ NAIC, Arecibo Observatory, HC03 Box 53995, PR 00612 \\ and \\ B. M. Gaensler \\ Harvard-Smithsonian Center for Astrophysics, 60 Garden Street MS-6, Cambridge, MA \\ 02138
}

\begin{abstract}
We present results from X-ray and radio observations of the recently discovered young Vela-like pulsar PSR J2021+3651, which is coincident with the EGRET $\gamma$-ray source GeV 2020+3658. A 19.0-ks Chandra ACIS-S observation has revealed a $\sim 20^{\prime \prime} \times 10^{\prime \prime}$ pulsar wind nebula that is reminiscent of the equatorial tori seen around some young pulsars, along with thermal emission from an embedded point source $\left(k T_{\infty}=0.15 \pm 0.02 \mathrm{keV}\right)$. We name the nebula G75.2+0.1. Its spectrum is well fit by an absorbed power-law model with photon index $\Gamma=1.7_{-0.2}^{+0.3}$, hydrogen column density $\mathrm{n}_{\mathrm{H}}=\left(7.8_{-1.4}^{+1.7}\right) \times 10^{21} \mathrm{~cm}^{-2}$, and an unabsorbed $0.3-10.0 \mathrm{keV}$ flux of $\left(1.9_{-0.3}^{+0.1}\right) \times 10^{-12} \mathrm{erg} \mathrm{cm}^{-2} \mathrm{~s}^{-1}$. We have spatially fit G75.2+0.1 with a model that assumes a toroidal morphology, and from this we infer that the torus is highly inclined $\left(83^{\circ} \pm 1^{\circ}\right)$ to the line of sight. A 20.8-ks Chandra observation in continuous-clocking mode reveals a possible
\end{abstract}

\footnotetext{
${ }^{1}$ Also Center for Space Research, M.I.T, Cambridge, MA 02139

${ }^{2}$ Eureka Scientific, 2452 Delmer St. Suite 100, Oakland, CA 94602-3017
} 
pulse detection, with a pulsed fraction of $\sim 37 \%$ and an H-test probability of occuring by chance of $1.2 \times 10^{-4}$. Timing observations with the Arecibo radio telescope spanning two years show that PSR J2021+3651 glitched sometime between MJDs 52616 and 52645 with parameters $\Delta \nu / \nu=(2.587 \pm 0.002) \times 10^{-6}$ and $\Delta \dot{\nu} / \dot{\nu}=(6.2 \pm 0.3) \times 10^{-3}$, similar to those of the largest glitches observed in the Vela pulsar. PSR J2021+3651 is heavily scattered $\left(\tau_{s c}=17.7 \pm 0.9 \mathrm{~ms}\right.$ at $1 \mathrm{GHz}$ ) and exhibits a significant amount of timing noise.

Subject headings: pulsars: general — pulsars: individual (PSR J2021+3651) — stars: neutron — gamma-rays: individual (GeV 2020+3658) — ISM: individual $(\mathrm{G} 75.2+0.1)$

\section{Introduction}

Pulsar wind nebulae (PWNe) allow us to study the basic energetic processes of young, rotation-powered pulsars. It is still unclear how the rotational kinetic energy of these objects is converted into a relativistic particle outflow. The Chandra X-ray Observatory's unprecedented spatial resolution has revealed that the winds from young, energetic pulsars can lead to highly structured PWNe. In some cases these PWNe take the form of an equatorial outflow with polar jets, where the best example of this is the Crab nebula (Weisskopf et al. 2000). These torus plus jet nebulae, of which there are roughly a half-dozen examples, are particularly interesting because a) they allow us to study the interaction of the PWN with its surrounding environment (the location of the bright torus is generally believed to mark the inner termination shock of the pulsar wind) and b) the well-defined morphology of the PWN can constrain the geometry of the pulsar. This is crucial for testing models of high-energy pulsations, such as the outer-gap model (e.g. Romani 1996) and the polar-cap model (e.g. Daugherty \& Harding 1996).

PSR J2021+3651 (spin period $P \sim 103.7 \mathrm{~ms}$, dispersion measure DM $\sim 369 \mathrm{pc} \mathrm{cm}^{-3}$, and flux density at $1400 \mathrm{MHz} S_{1400} \sim 100 \mu \mathrm{Jy}$ ) was discovered by Roberts et al. (2002) with the 305-m Arecibo radio telescope during a targeted search for radio pulsations from X-ray sources proposed as counterparts to unidentified EGRET $\gamma$-ray sources (Roberts, Romani, \& Kawai 2001). Timing observations of PSR J2021+3651 revealed that although it is dim, it is young and energetic (characteristic age $\tau_{c} \equiv P / 2 \dot{P}=17 \mathrm{kyr}, \dot{E} \equiv 4 \pi^{2} I \dot{P} / P^{3}=3.6 \times 10^{36}$ ergs $\mathrm{s}^{-1}$, assuming moment of inertia $I=10^{45} \mathrm{~g} \mathrm{~cm}^{-2}$ ), and a likely counterpart to the $A S C A \mathrm{X}-$ ray source AX J2021.1+3651 and the EGRET $\gamma$-ray source GeV J2020+3658. This discovery bolsters the idea that many of the Galactic unidentified EGRET sources (see Hartman et al. 1999) are young energetic pulsars that were not detected in large-scale radio pulsar surveys. 
While the spin properties of PSR J2021+3651 are very similar to those of the known $\gamma$ ray pulsars Vela (PSR B0833-45, $P=89.3 \mathrm{~ms}, \dot{E}=6.9 \times 10^{36} \mathrm{ergs} \mathrm{s}^{-1}$ ) and PSR B1706-44

$\left(P=102.5 \mathrm{~ms}, \dot{E}=3.4 \times 10^{36} \mathrm{ergs} \mathrm{s}^{-1}\right.$ ), the $12 \mathrm{kpc}$ distance inferred from its DM (which places it on the far edge of the outer spiral arm) using the NE2001 electron model of the Galaxy (Cordes \& Lazio 2002) would make it much more $\gamma$-ray efficient than either Vela or PSR B1706-44. In fact, it would have to be even more $\gamma$-ray efficient than the much-less energetic $\gamma$-ray pulsars Geminga and PSR B1055-52 (assuming the NE2001 DM distance to PSR B1055-52 of $0.72 \mathrm{kpc}$ ). This is contrary to the observation that $\gamma$-ray efficiency $\eta_{\gamma} \equiv L_{\gamma} / \dot{E}$ increases with age (Thompson et al. 1999) and the theoretical expectation that it is inversely proportional to the square-root of the spin-down luminosity $\left(\eta_{\gamma} \propto \dot{E}^{-1 / 2}\right.$, e.g. Zhang \& Harding 2000). However, since DM distances can be off by a factor of a few, PSR J2021+3651 may be significantly closer (or farther) than its DM implies.

The similarity of PSR J2021+3651 to the Vela pulsar, which is surrounded by a highlystructured pulsar wind nebula (see Helfand, Gotthelf, \& Halpern 2001), prompted us to make X-ray observations of the area surrounding PSR J2021+3651. Only $\sim 30$ of the $>1300$ known pulsars in the Galaxy are Vela-like (i.e. with $P \sim 100 \mathrm{~ms}, \dot{E} \sim 10^{36}-10^{37} \mathrm{ergs} \mathrm{s}^{-1}$, and characteristic ages $\tau_{c} \sim 10-20 \mathrm{kyr}$, see Kramer et al. 2003). Half of these are known to have associated PWN (Kaspi, Roberts, \& Harding 2004). Here we present the results of X-ray observations made with the Chandra ACIS-S detector. These observations resolve the $A S C A$ source into a new PWN, which we name G75.2+0.1, and a thermally emitting point source, likely the neutron-star surface. Chandra observations in continuous-clocking mode show a potential detection of X-ray pulsations from PSR J2021+3651. We also present radio timing observations made with the Arecibo telescope, which show that PSR J2021+3651 has glitched and is highly scattered.

\section{X-ray Observations}

\subsection{X-ray Imaging}

We made Chandra observations with the ACIS-S detector in VFAINT mode on 2003 February 12 to image PSR J2021+3651 and its surrounding region. To reduce CCD photon pile-up from a potentially bright point source, a quarter sub-array was used. These data were analyzed using $\mathrm{CIAO}^{1}$ version 3.0.1 and CALDB 2.3. Filtering for good time intervals and accounting for dead-time resulted in a total integration time of $19.0 \mathrm{ks}$. We subtracted

\footnotetext{
${ }^{1}$ see http://cxc.harvard.edu/ciao
} 
particle background from the image using a 100-ks combination of two ACIS-stowed images from 2002 Sept and $2003 \mathrm{May}^{2}$ and then corrected for the different exposure over the chip. The resulting image is shown in Figure 1.

This image reveals a point source embedded in an axisymmetric inner nebula $\sim 20^{\prime \prime} \times 10^{\prime \prime}$ across, surrounded by fainter diffuse emission. We refer to this obvious PWN as G75.2+0.1. The point source is at right ascension $20^{h} 21^{m} 05^{s} .46$ and declination $+36^{\circ} 51^{\prime} 04^{\prime \prime} .8$ (J2000). We estimate a positional error of $\sim 1^{\prime \prime}$ by comparing the position of another bright X-ray point source on the S3 chip with a catalogued optical counterpart. Subtracting the nebular background, the point source has a count rate of $0.0094 \pm 0.0009$ counts s$^{-1}$ and the nebula has a background-subtracted count rate of $0.075 \pm 0.004$ counts s$^{-1}$ in the $0.3-10.0 \mathrm{keV}$ range.

The greyscale of Figure 1 shows the image smoothed with a 1.2" (FWHM) gaussian to bring out the fine structure of the inner nebula. The white contours indicate the position of the point source and are shown to distinguish it from the bright bar running along the axis of the nebula. This bar is the most obvious resolved feature of the nebula. Also overlaid are black contours of the same image smoothed with a $3.5^{\prime \prime}$ gaussian and scaled to bring out what are possibly faint jets along the axis of the nebula. We estimate $62 \pm 32$ counts may be coming from these putative jets. The nebula appears symmetric about the minor axis, but the point source is clearly offset from the major axis of the nebula. The overall morphology is suggestive of the torus plus weak jet morphologies of the Crab and (likely) Vela nebulae (Weisskopf et al. 2000; Helfand et al. 2001). We discuss the geometry of the PWN further in $\S 4.4$.

\subsection{X-ray Spectroscopy}

The spectra of the PWN and point source can be measured individually using Chandra's excellent spatial resolution. We fit the spectrum of the nebula and point source using CIAO's Sherpa environment (version 3.0.1) and XSPEC (version 11.2.0). To isolate the nebula, we used a circular annulus centered on the point source and extending from a radius of 1.6-100 pixels $\left(0.492^{\prime \prime} \times 0.492^{\prime \prime}\right.$ per pixel $)$ and a circular region from an apparently flux-free area of the S3 chip to estimate the background. We find that the nebula has a hard spectrum (see Figure 2) that is well fit (reduced $\chi_{\nu}{ }^{2}=1.1$ for 27 degrees of freedom) by an absorbed power-law model with hydrogen column density $\mathrm{n}_{\mathrm{H}}=\left(7.8_{-1.4}^{+1.7}\right) \times 10^{21} \mathrm{~cm}^{-2}$, photon index $\Gamma=1.7_{-0.2}^{+0.3}$, and a $0.3-10 \mathrm{keV}$ absorbed flux of $(1.2 \pm 0.1) \times 10^{-12} \mathrm{erg} \mathrm{cm}^{-2} \mathrm{~s}^{-1}(90 \%$ confidence intervals). The best-fit black-body model gives reduced $\chi_{\nu}{ }^{2}=2.3$ for 27 degrees

\footnotetext{
${ }^{2}$ see http://cxc.harvard.edu/contrib/maxim/acisbg
} 
of freedom, a comparatively poor fit to the data.

To isolate the point source, we used a circular extraction region of radius 1.6 pixels, and a surrounding annulus of width 1.5 pixels to subtract the nebular background. Because the energy dependent PSF extends significantly past 1.6 pixels at this location, we corrected for this by using "mkpsf" to generate model PSFs at several energies between $0.5-6 \mathrm{keV}$ from which we estimated the source minus background enclosed energy fraction. We then fit a linear function to these points which we used to correct the effective area model used in the spectral fit. Based on the observed count rate, we estimate a negligible pile-up fraction of $\sim 0.6 \%$ using HEASARC's webPIMMs tool ${ }^{3}$. We find that there is very little emission from the point source above $3 \mathrm{keV}$. Its spectrum is adequately fit (reduced $\chi_{\nu}{ }^{2}=1.7$ for 9 degrees of freedom) by an absorbed black-body model, where we have fixed $n_{H}$ to the value obtained by fitting the PWN's spectrum. The best-fit black-body model and $90 \%$ confidence region is $k T_{\infty}=0.15 \pm 0.02 \mathrm{keV}$ with an absorbed flux of $(2.8 \pm 0.2) \times 10^{-14} \mathrm{erg}$ $\mathrm{cm}^{-2} \mathrm{~s}^{-1}(0.3-10.0 \mathrm{keV})$. Since $k T_{\infty}$ and $\mathrm{n}_{\mathrm{H}}$ are highly covariant, we also made a joint fit of an absorbed black body for the pulsar and an absorbed power law for the nebula, tying the $\mathrm{n}_{\mathrm{H}}$ of both models together. This gave values and errors that were essentially the same as those from the individual fits. Since we expect non-thermal emission from the magnetosphere of the pulsar, we also fit a black-body plus power-law model to the point source, fixing the photon index at a typical value of 1.5. This did not improved the fit (reduced $\chi_{\nu}{ }^{2}=1.8$ for 7 degrees of freedom), but the temperature and flux of the black-body component were left relatively unchanged. This suggests a maximum non-thermal absorbed flux of $\sim 3 \times 10^{-14}$ erg $\mathrm{cm}^{-2} \mathrm{~s}^{-1}(0.3-10.0 \mathrm{keV})$.

The total flux measured here is a factor of four smaller than seen by $A S C A$ (Roberts et al. 2001; Roberts et al. 2002). We note that the $A S C A$ extraction region has a radius of $\sim 4^{\prime}$, and thus includes areas well outside the $2^{\prime}$-wide field of view of the quarter array used here. A highly smoothed image of the Chandra data suggests there may be additional diffuse emission that could extend well beyond the chip. Furthermore, examination of the $A S C A$ image suggests there may be additional point sources or structures within the $A S C A$ extraction region that are not within the Chandra field of view. However, despite the flux discrepancy, the derived $\mathrm{n}_{\mathrm{H}}$, nebular photon index and point source $k T_{\infty}$ are consistent with the $A S C A$ measurements of the source.

\footnotetext{
${ }^{3}$ see http://heasarc.gsfc.nasa.gov/Tools/w3pimms.html
} 


\subsection{X-ray Pulsations}

To search for X-ray pulsations from PSR J2021+3651, we also obtained Chandra ACIS$\mathrm{S}$ data in continuous-clocking mode, which provides an effective time resolution of $2.85 \mathrm{~ms}$ by sacrificing one dimension of spatial resolution. The roll angle of the telescope placed the 1 dimensional image at an angle of $\sim 62^{\circ}(\mathrm{N}-\mathrm{E})$. The events were filtered for good time intervals and periods of high background, leaving $20.8 \mathrm{ks}$ of integration time. We corrected the read-out times to intrumental times of arrival by removing telescope dither, motion of the detector with respect to the telescope, and the average lag between the readout time and the true times of arrival of the photons. The events were then barycentered using the JPL DE405 ephemeris (Standish 1998). Given prior knowledge of the point-source spectrum from our imaging observations, we produced an event list with a $0.5-3 \mathrm{keV}$ energy cut and a 3-pixel-radius extraction region. We also produced event lists for a number of other energy cuts and a range of extraction regions and then dithered the events by adding random deviates in the range $0-2.85 \mathrm{~ms}$ to remove windowing effects. The event lists were folded at the predicted spin frequency of $9.64094861 \mathrm{~Hz}$ according to the contemporaneous radio timing ephemeris (see $§ 3$ ), which revealed a likely periodic signal (see Figure 3 ). The signal shows up for a range of extraction region sizes and energy cuts, but has a maximum signal strength for an extraction region of radius 3 pixels with a $0.5-3 \mathrm{keV}$ energy cut. Performing a variant of the H-test (De Jager et al. 1989), using unbinned events, we find an H-test score of $h \sim 11.4$, which corresponds to a chance probability of $1.2 \times 10^{-4}$ for a single trial. This has an equivalent Gaussian sigma of $3.7 \sigma$. Searching to a maximum harmonic of 20, the H-test finds the largest significance when two harmonics are summed.

Although this signal is not a certain detection of pulsations from PSR J2021+3651, it is intriguing. If real, these pulsations indicate a pulsed fraction of $\sim 37 \%$, where we define pulse fraction to be the ratio of the number of counts above the 1- $\sigma$ upper limit on the lowest bin in the light curve (see Figure 3) to the total number of background-subtracted counts (in this case, most of the background is from the nebula). From the point source count-rate of our imaging observations, we estimate that $\sim 190$ of the counts in the light curve are from the pulsar and that the rest are from the nebula and other background. We discuss the pulse shape in $\S 4.2$.

\section{Radio Timing}

Since the discovery of PSR J2021+3651 in 2002 February, we have been making roughly monthly radio timing observations of the pulsar at $20 \mathrm{~cm}$ using the Arecibo radio telescope and multiple Wideband Arecibo Pulsar Processor digital correlator backends (WAPPs, for 
details see Dowd, Sisk, \& Hagen 2000). Each WAPP was configured for $100 \mathrm{MHz}$ of bandwidth, with 512 lags and $200 \mu$ s samples. Whenever possible, we used three WAPP backends, typically centered at 1170,1420 , and $1520 \mathrm{MHz}$. PSR J2021+3651 is significantly scattered (see Figure 4). Fitting the profiles with a gaussian convolved with a one-sided exponential $\left(e^{-t / \tau_{s c}}\right)$ we find a frequency dependence of $\tau_{s c}$ that is well fit by a power law of index $-4.2 \pm 0.6$. This is consistent with a pure Komolgorov spectrum (i.e. $\tau_{s c} \propto \nu^{-4.4}$, see Rickett 1977). Using the measured frequency dependence, we find $\tau_{s c}=17.7 \pm 0.9 \mathrm{~ms}$ at $1 \mathrm{GHz}(90 \%$ confidence).

Pulse profiles from each epoch were cross-correlated with a representative high signalto-noise template to generate times of arrival (TOAs). Using TEMPO ${ }^{4}$ we fit these TOAs to a multi-parameter rotational model of the pulsar. The position of the pulsar was fixed at the Chandra X-ray position. Our timing analysis reveals that a glitch occured between MJDs 52616 and 52645 . We can connect phase across it by fitting an instantaneous jump in frequency and frequency derivative $\left(\Delta \nu / \nu=2.587 \pm 0.002 \times 10^{-6}\right.$ and $\Delta \dot{\nu} / \dot{\nu}=6.2 \pm$ $0.3 \times 10^{-3}$ ), which are comparable to those seen in the largest glitches of the Vela pulsar (Dodson et al. 2002). There is only marginal evidence for a glitch decay in the data, which is not surprising given that the typical timescale for such a decay is on the order of days to weeks (Dodson et al. 2002) and is therefore not resolved by our sparse monitoring. The preand post-glitch rotational ephemerides of PSR J2021+3651 and the complete set of glitch parameters are presented in Table 1, along with derived quantities.

The post-fit timing residuals show significant systematics when only the frequency and first frequency derivative are fit (see Figure 5). We find that the residuals can be rendered featureless by including a frequency second and third derivative into the fit. Alternately, the residuals can be rendered featureless by allowing TEMPO to fit for position. However, the pre-, post-glitch, and Chandra positions are inconsistent, suggesting that timing noise dominates the residuals rather than an incorrect position.

\section{Discussion}

\subsection{Distance}

As discussed in Roberts et al. (2002), the DM distance of PSR J2021+3651 is quite large ( 12 kpc using the NE2001 electron density model of the galaxy, see Cordes \& Lazio 2002) and would place PSR J2021+3651 on the outer edge of the outer spiral arm of the

\footnotetext{
${ }^{4}$ see http://www.pulsar.princeton.edu/tempo
} 
Galaxy. This large distance leads to a very high inferred $\gamma$-ray efficiency $\left(\eta_{\gamma} \equiv L_{\gamma} / \dot{E} \sim 0.18\right.$ in the $100 \mathrm{MeV}-10 \mathrm{GeV}$ range, assuming 1-sr beaming). Cordes \& Lazio (2002) note that the electron density model is seldom off by more than $50 \%$ when predicting distances, except when the line of sight intersects a particularly dense scattering screen. We find a scattering measure $\mathrm{SM}=(1.5 \pm 0.1) d_{10}^{-5 / 6} \mathrm{kpc} \mathrm{m}^{-20 / 3}$ towards PSR J2021+3651, where $\tau_{s c} \equiv 1.10 \mathrm{~ms}$ $\mathrm{SM}^{6 / 5} \nu^{-22 / 5} d$ (Cordes \& Lazio 2002), that is two orders of magnitude larger than predicted by the NE2001 model (although PSR J2021+3651 is in the direction of the Cygnus region and pulsars in that direction are often highly scattered). This suggests the presence of a clump of scattering material along the line of sight not accounted for by the NE2001 model. However, small clumps of material that only change the DM by $10-20 \mathrm{pc} \mathrm{cm}{ }^{-3}(<5 \%$ in the case of PSR J2021+3651) can change the SM by a factor of a hundred. Hence, the large SM does not by itself imply a significantly closer distance. In order for the distance to imply a much smaller $\gamma$-ray efficiency, a significant source of dispersion $\left(\sim 200 \mathrm{pc} \mathrm{cm}^{-3}\right)$ unaccounted for by the model is necessary. Such a DM enhancement could come from an ultra-compact HII region or an OB association. An examination of the Midcourse Space Experiment $8.3 \mu \mathrm{m}$ IR data (available from the NASA/IPAC Infrared Science Archive) and preliminary radio imaging with VLA data we have obtained (work in preparation) show no evidence for an ultra-compact HiI region. The OB association Cyg 1,8,9 lies in the direction of PSR J2021+3651(Yadigaroglu \& Romani 1997; Mel'nik \& Efremov 1995), however there is currently no evidence that this is significantly contributing to the amount of ionized gas along the line of sight or that the pulsar was born in this association. Since the DM distance places PSR J2021+3651 at the very outer edge of the Galaxy one would expect our spectral fit to give an $\mathrm{n}_{\mathrm{H}}$ consistent with the total column density in this direction. The X-ray absorption found here, $\mathrm{n}_{\mathrm{H}}=\left(7.8_{-1.4}^{+1.7}\right) \times 10^{21} \mathrm{~cm}^{-2}$, is lower than the total Galactic Hi column density in the direction of PSR J2021+3651, $1.2 \times 10^{22} \mathrm{~cm}^{-2}$, estimated using the FTOOL $n h^{5}$ (which uses the Hi map of Dickey \& Lockman 1990). This argues for a distance somewhat closer than the predicted DM distance, but not closer by factor of a few. Hence a distance of $\sim 8 \mathrm{kpc}$ is quite plausible, but a distance of less than $5 \mathrm{kpc}$, as required to make the $\gamma$-ray efficiency similar to that of PSR B1706-44 $\left(\eta_{\gamma}=0.01\right.$, see Roberts 2003), is hard to justify given current observations of the region. We adopt a nominal distance of $10 \mathrm{kpc}$ to use in scaling quantities $\left(d_{10} \equiv d / 10 \mathrm{kpc}\right)$ that depend on distance.

\footnotetext{
${ }^{5}$ see http://heasarc.gsfc.nasa.gov
} 


\subsection{Neutron Star Thermal Emission and Pulsations}

We find a black-body temperature of $k T_{\infty}=0.15 \pm 0.02 \mathrm{keV}$ for the point source, similar to that found for the Vela pulsar $\left(k T_{\infty}=0.128 \pm 0.003 \mathrm{keV}\right.$; Pavlov et al. 2001) and PSR B1706-44 $\left(k T_{\infty}=0.14 \pm 0.02 \mathrm{keV}\right.$; Gotthelf et al. 2002). Accounting for the effects of gravitational redshift, this corresponds to a surface temperature $k T_{s}=0.19 \pm 0.03 \mathrm{keV}$ for the canonical neutron-star radius of $10 \mathrm{~km}$ and mass of $1.4 \mathrm{M}_{\odot}$. The point source has an unabsorbed flux $F_{\mathrm{X}}=6.3 \times 10^{-13} \mathrm{erg} \mathrm{cm}^{-2} \mathrm{~s}^{-1}$ in the range $0.3-10.0 \mathrm{keV}$. Scaling the luminosity to a distance of $10 \mathrm{kpc}, L_{\mathrm{X}}=7.5 d_{10}^{2} \times 10^{33} \mathrm{erg} \mathrm{s}^{-1}(0.3-10.0 \mathrm{keV})$. One can calculate the ratio of the black-body emission radius $R_{e}$ and the distance to the source using the bolometric flux $7.8 \times 10^{-13} \mathrm{erg} \mathrm{cm}^{-2} \mathrm{~s}^{-1}$ and the Stefan-Boltzman law. Correcting for gravitational redshift, this gives $R_{10} / d_{10}=0.3$, where $R_{10} \equiv R_{e} / 10 \mathrm{~km}$. For any reasonable distance, the simple black-body model suggests that the emission is coming from hot spots on the surface, rather than from the entire surface. This is not unreasonable, as some high-energy emission models predict hot spots due to back-flowing particles.

Using the non-magnetized neutron-star hydrogen and iron atmosphere models of Gaensicke et al. (2002), which assume a neutron-star radius of $10 \mathrm{~km}$ and a mass of $1.4 \mathrm{M}_{\odot}$, changes the temperature significantly. Fixing the $\mathrm{n}_{\mathrm{H}}$ to the value derived from the nebular fit, for a hydrogen atmosphere we get a best-fit value of $k T_{s}=0.06 \pm 0.01 \mathrm{keV}$ (which is similar to the hydrogen atmosphere fit of the Vela pulsar, $k T_{\infty}=0.059 \pm 0.003 \mathrm{keV}$; Pavlov et al. 2001) and a flux of $2.9 \times 10^{-14} \mathrm{erg} \mathrm{cm}^{-2} \mathrm{~s}^{-1}(0.3-10.0 \mathrm{keV})$. However, the normalization from this fit implies a $\sim 0.8 \mathrm{kpc}$ distance, which is unreasonable. Again fixing the $\mathrm{n}_{\mathrm{H}}$ to the nebular value, for an iron atmosphere model we get a best-fit value of $k T_{s}=0.22 \pm 0.01 \mathrm{keV}$ and a flux of $3.6 \times 10^{-14} \mathrm{erg} \mathrm{cm}^{-2} \mathrm{~s}^{-1}(0.3-10.0 \mathrm{keV})$. The implied distance here is $\sim 13 \mathrm{kpc}$, which is roughly consistent with the emission coming from the entire surface.

Based on the soft spectrum of the point source, one would expect pulsations to be due primarily to thermal emission. The profile found by folding the X-ray events of our continuous-clocking data with the radio ephemeris shows a somewhat narrow (duty cycle $\sim 0.3$ ) peak and interpulse, which is possible for thermal pulsations given the right geometry. If we are seeing emission from hot spots at both poles then it is possible to get two sinusoidal peaks separated by a plateau of emission (Beloborodov 2002). In fact, the geometry inferred from the PWN (see $\S 4.4$ ) is consistent with the pulse profile seen here. As noted in $\S 2.2$, a non-thermal component is also allowed by the spectrum. The possible pulsations might be a combination of pulsed thermal and magnetospheric emission, but the number of pulsed counts $(\sim 69)$ is too large to consist solely of magnetospheric emission. All the known $\gamma$-ray pulsars have also been detected as X-ray pulsars with non-thermal emission components to their spectra (Kaspi et al. 2004). We note that a lack of X-ray pulsations would not preclude 
PSR J2021+3651 from being a $\gamma$-ray pulsar. For instance, X-ray pulsations in PSR B1706-44 are relatively difficult to detect even though PSR B1706-44 is a well-established $\gamma$-ray pulsar. Thus, PSR J2021+3651 remains an excellent $\gamma$-ray pulsar candidate.

\subsection{PWN Spectrum}

We found that the spectrum of the nebula is well fit by an absorbed power-law model with $\mathrm{n}_{\mathrm{H}}=\left(7.8_{-1.4}^{+1.7}\right) \times 10^{21} \mathrm{~cm}^{-2}$ and $\Gamma=1.7_{-0.2}^{+0.3}$. The photon index $\Gamma$ is roughly consistent with what is observed from the PWN of other similar pulsars like Vela $(\Gamma=1.50 \pm 0.04 ;)$ and PSR B1706-44 $\left(\Gamma=1.34_{-0.30}^{+0.24}\right.$; Gotthelf et al. 2002), although it is somewhat higher. The relationship of Gotthelf (2003) between the photon index of the nebula and the spin-down energy of the pulsar (equation 3 of that paper) gives $\Gamma=1.22 \pm 0.33$, which is marginally consistent with the $\Gamma$ measured here.

The unabsorbed nebular flux of G75.2+0.1 is $F_{\mathrm{X}}=\left(1.9_{-0.3}^{+0.1}\right) \times 10^{-12} \mathrm{erg} \mathrm{cm}^{-2} \mathrm{~s}^{-1}$ in the $0.3-10.0 \mathrm{keV}$ range. This corresponds to an $\mathrm{X}$-ray luminosity in the same energy range of $L_{\mathrm{X}}=\left(2.3 d_{10}{ }^{2}\right) \times 10^{34} \mathrm{erg} \mathrm{s}^{-1}$. For the same energy range, we calculate an X-ray efficiency $\left(\eta_{\mathrm{X}} \equiv L_{\mathrm{X}} / \dot{E}\right)$ of $0.009 d_{10}{ }^{2}$ from the combined point-source and nebular luminosities. This value fits well with the claimed relationship between the spin-down energy of a pulsar and its nebular X-ray efficiency (c.f. Possenti et al. 2002; Chevalier 2000) even if the distance is as close as $3 \mathrm{kpc}$. However, note that this is only for the bright inner nebula. If there is significantly more flux coming from diffuse emission (as suggested by the $A S C A$ data and in analogy with the Vela nebula, see below) then the efficiency will be higher.

\subsection{PWN Size and Morphology}

The generally accepted view of young PWNe (see Kaspi et al. 2004, and references therein) is that they are synchrotron bubbles blown at the center of an expanding supernova remnant (SNR). High-energy electrons and positrons, along with magnetic field, are continuously injected into the bubble by the pulsar. Given that the characteristic age of PSR J2021+3651 is much larger than the typical time scale for a SNR entering the Sedov phase, it is likely (although not certain since characteristic ages can be off by an order of magnitude or more) that the outer edge of the bubble (at radius $R_{P}$ ) is expanding subsonically into the shocked supernova ejecta. The bubble is bounded at the inner edge (at radius $R_{T}$ ) by the termination of the ultra-relativistic pulsar wind.

The physical size of G75.2+0.1's inner nebula is $\sim(0.5 \times 1) d_{10}$ pc. As PSR J2021+3651's 
spin-down energy is within a factor of two of that of the Vela pulsar, one might naively expect the sizes and morphologies of their nebulae to be comparable. Scaling the $\sim 10^{\prime \prime}$ radius of G75.2+0.1's inner nebula to that of the Vela pulsar's inner nebula, which has a radius of $52 . " 7$ (Helfand et al. 2001) and whose distance is well known (290 pc, Dodson et al. 2003), places G75.2+0.1 at a distance of $\sim 1.5 \mathrm{kpc}$, which seems unreasonable given the DM. In fact, G75.2+0.1 would have to be a few times larger than the Vela nebula to put it at a distance that seems at all reasonable based on its DM. However, since the Vela inner nebula is only the central part of a much larger and more luminous nebula, a better comparision between the sizes of these two objects could be made if the true extent of G75.2+0.1 is determined in a deeper observation with a larger field of view.

The bright inner nebula of Vela and G75.2+0.1 may be directly downstream of the termination shock of the relativistic pulsar wind. The location of the termination shock depends primarily on the energy output of the pulsar and the pressure in the nebula. The pressure in the nebula depends upon the total energy input during the pulsar's lifetime and the losses due to adiabatic and synchrotron processes (possibly complicated by the passage of the SNR reverse shock). The total energy input is highly dependent on the initial spin period, which is unknown and could range from $\sim 10-100 \mathrm{~ms}$ (Kaspi et al. 2001; Migliazzo et al. 2002). We currently do not know the full extent of G75.2+0.1, which is likely much larger than the inner nebula seen here. While it is true that the pressure in the PWN should be balanced by the pressure in the surrounding SNR, we know neither the age (as the characteristic age is a poor estimate of the true age for young pulsars) nor the size (since we have not observed a shell) of the potential remnant. Therefore, although the Vela pulsar is twice as energetic as PSR J2021+3651, it is entirely possible that G75.2+0.1 is several times larger than the Vela nebula.

The size of the torus places an upper limit on the termination shock radius $R_{T}$. In analogy to Eqn. 1 in Roberts et al. (2003) (see also Chevalier 2000) we can estimate the post-shock magnetic field to be

$$
B_{N} \sim 2 \times 10^{-5}\left(\frac{\epsilon_{t}^{1 / 2}}{f_{t}\left(R_{T} / r\right) d_{10}}\right) \mathrm{G}
$$

where $r$ is the measured location of the torus, $\epsilon_{t} \lesssim 1$ is the ratio of the magnetic energy to the total energy in the post-shock flow and $f_{t} \lesssim 1$ is a geometrical factor allowing for nonspherical outflow. Note that the PWN of PSR B1706-44 (which has a virtually identical $\dot{E}$ to PSR J2021+3651) is both much fainter and smaller than G75.2+0.1. This implies that the magnetic field in PSR B1706-44's nebula is smaller but the density of synchrotron emitting particles is larger. PSR B1706-44 is a known TeV source (Kifune et al. 1995), this suggests PSR J2021+3651 may be an excellent target for ground-based Cerenkov detectors. 
Our G75.2+0.1 image is rather sparse and Poisson-limited, and so a unique model for its morphology cannot be defined. Nevertheless, the axisymmetry of G75.2+0.1 suggests that the morphology may be best described as that of a torus, with possibly weak jets directed along the axis of symmetry (see Figure 1). To investigate the question of G75.2+0.1's morphology quantitatively, we spatially fit the PWN using the relativistic torus fitting described in Ng \& Romani (2004). Since G75.2+0.1 appears to have a double ridge, our fiducial model is similar to that of the Vela pulsar PWN: twin tori of radius $r$, symmetrically spaced by distance $d$ on either side of the pulsar spin equator. The torus axis is at position angle $\Psi$ $(\mathrm{N}-\mathrm{E})$ and inclination $\zeta$ to the line of sight. The bulk flow velocity down-stream from the termination shock is $\beta_{T}$ and a 'blur' parameter $\delta$ is applied to account for the unmodeled post-shock synchrotron cooling length. A uniform background and a detailed point source model computed for the pulsar spectrum and chip position are also included. The 3-D model is projected to the plane of the sky, a Poisson-based figure of merit is minimized to find the best-fit parameters and the statistical errors on these parameters are estimated by refitting many Poisson realizations of the best-fit model, each with the same number of counts as seen in the data.

The derived parameters from the fit are summarized in Table 2. The errors quoted are statistical 1- $\sigma$ values only. Systematic errors due to unmodeled features are difficult to quantify. One estimate of their magnitude can be made by excluding an elliptical zone about the pulsar, extended along the symmetry axis where an unmodeled polar jet might be present. Re-fitting the model to the surrounding data, we obtain values within $1.5 \sigma$ of the original fit for all parameters, except $\beta_{T}$ which now is $0.64 \pm 0.02 ; \mathrm{Ng} \&$ Romani (2004) note that for this parameter the statistical errors tend to be small and dominated by systematic uncertainties for edge-on tori. In this particular case, the value of $\beta_{T}$ is likely being dominated by the bright clump to the south-west of the pulsar. We have also fit a single torus; again the geometrical parameters are quite similar, although a larger blur parameter $\delta=1.8^{\prime \prime}$ is required to cover the broader torus. The fit statistic for the model is of course larger than that of the 2-torus model, by an amount similar to that corresponding to $1-\sigma$ excursions in the individual fit parameters. Unfortunately, this does not alone show that the double torus is a significantly better model; we cannot apply an F-test, since the fit statistic is not $\chi^{2}$.

Clearly a deeper image is needed to draw firm conclusions, but we can discuss the geometrical implications of the fit angles. The large $\zeta$ implies that we are viewing the pulsar near the spin equator. In the outer magnetosphere picture of Romani \& Yadigaroglu (1995) this is indeed where we expect to see $\gamma$-ray emission. More particularly, Figure 4 of that paper shows that for $\zeta \approx 83^{\circ}$ and magnetic impact angle $\beta$ modest (to allow detection of the radio emission), we expect two $\gamma$-ray pulses separated by $\sim 180^{\circ}$, with the first lagging the 
radio peak by $\sim 10^{\circ}$. Intriguingly, McLaughlin \& Cordes (2003) found apparently significant pulsations folding the $\gamma$-ray photons from this source near the extrapolated $P$ for two viewing periods; the light curves show two narrow peaks separated by 0.5 in phase. It is important to note that for $\zeta$ near $90^{\circ}$, such a light curve is also naturally expected in the two pole model of Dyks \& Rudak (2003) and may be possible in polar-cap models (Daugherty \& Harding 1996). The separation of the two tori suggest bright emission at spin axis angles $\theta_{T}=\tan ^{-1}(2 r \sin \zeta / d)=77^{\circ}, 103^{\circ}$, as viewed from the pulsar. If these represent enhanced $\mathrm{e}^{ \pm}$flowing radially from near the magnetic poles then we might infer a magnetic inclination $\alpha \approx 77^{\circ}$. This would imply an impact parameter $\beta=\zeta-\alpha \approx+6^{\circ}$. Polarization sweep measurements could test this picture. The unscattered pulse half-power width $\sim 33^{\circ}$ of PSR J2021+3651 is substantially wider than the $2.5^{\circ}(P / 1 \mathrm{~s})^{-1 / 2} / \sin \alpha$ width expected for a core component, but is similar to the $5.8^{\circ}(P / 1 \mathrm{~s})^{-1 / 2}$ width for an outer cone (Rankin 1993). Of all the fit geometrical parameters, the most robust is the fit PA of the symmetry axis $\Psi$. Although difficult to measure, given the large DM and expected substantial Faraday rotation, the absolute position angle at the polarization sweep maximum would provide an interesting check of the inference that this represents the pulsar spin axis.

\section{Conclusions and Further Work}

We have used the Chandra X-ray Observatory to observe the young and energetic pulsar PSR J2021+3651 and have detected a new PWN, G75.2+0.1, as well as an embedded point source near the radio timing position of the pulsar. The morphology of G75.2+0.1 is perhaps best decribed as an equatorial torus, and we have derived geometrical parameters for the orientation of the system by spatially fitting the nebula. Spectral fitting of the nebula shows that it is similar to the nebulae of other energetically-similar pulsars. Spectral fitting of the point source shows that it is well fit by an absorbed black-body model or a black-body plus power-law model. Radio timing observations of PSR J2021+3651 have revealed a glitch similar to the largest glitches seen in the Vela pulsar. Analysis of countinuous-clocking data from Chandra indicates that there may be weak X-ray pulsations from the source (pulsed fraction of $\sim 37 \%$ ) at the period predicted by the radio ephemeris. PSR J2021+3651 remains the most likely counterpart to the high-energy EGRET $\gamma$-ray source GeV J2020+3651 (which is the 10 th brightest $\gamma$-ray source above $1 \mathrm{GeV}$ ). It is an excellent $\gamma$-ray pulsar candidate for the AGILE and GLAST missions, although a contemporaneous ephemeris will be essential, given the large amount of timing noise the pulsar exhibits.

Deeper observations with Chandra and XMM-Newton are needed to clarify the morphol-

ogy of G75.2+0.1 and to confirm the presence of pulsations. Such observations are also able 
to confirm whether the faint outer jet hinted at here is real. Currently, there only about a half dozen PWNe with torus plus jet morphologies, with the Crab and (likely) Vela nebulae as the best examples. With a deeper observation, G75.2+0.1 may prove to be the third best example. Further work by our group will also include analysis of radio polarization data we have taken with Arecibo. These data may help bolster the geometrical interpretation described here if the swing of the polarization angle across the pulse is measurable. Proper-motion measurements could check if PSR J2021+3651 is moving along the axis of the nebula, which is the case for both Vela and the Crab. However, measuring the proper motion of PSR J2021+3651 (which will likely be small since the distance is likely large) will be very difficult both through radio timing because of timing noise or through VLBI because the radio source is very faint. We are also analyzing VLA observations (in A, C, and D arrays) of the source region to look for a radio PWN and/or SNR. This is important for characterizing the surrounding medium, which has an important effect on the morphology of the PWN and its size.

J.W.T.H. is an NSERC PGS A fellow. V.M.K. gratefully acknowledges support from NSERC Discovery Grant 228738-03, NSERC Steacie Supplement 268264-03, a Canada Foundation for Innovation New Opportunities Grant, FQRNT Team and Centre Grants, and NASA Long-Term Space Astrophysics Grant NAG5-8063. V.M.K. is a Canada Research Chair and Steacie Fellow. R.W.R. acknowledges support from NASA grants NAGS-13344 and SAOG03-4093B. The Arecibo Observatory is part of the National Astronomy and Ionosphere Center, which is operated by Cornell University under a cooperative agreement with the National Science Foundation.

\section{REFERENCES}

Beloborodov, A. M. 2002, ApJ, 566, L85

Chevalier, R. A. 2000, ApJ, 539, L45

Cordes, J. M. \& Lazio, T. J. W. 2002, ApJ, submitted, http://xxx.lanl.gov/abs/astro$\mathrm{ph} / 0007310$

Daugherty, J. K. \& Harding, A. K. 1996, ApJ, 458, 278

De Jager, O. C., Swanepoel, J. W. H., \& Raubenheimer, B. C. 1989, A\&A, 221, 180

Dickey, J. M. \& Lockman, F. J. 1990, ARAA, 28, 215 
Dodson, R. G., McCulloch, P. M., \& Lewis, D. R. 2002, ApJ, 564, L85

Dowd, A., Sisk, W., \& Hagen, J. 2000, in ASP Conf. Ser. 202: IAU Colloq. 177: Pulsar Astronomy - 2000 and Beyond, 275

Dyks, J. \& Rudak, B. 2003, ApJ, 598, 1201

Gaensicke, B. T., Braje, T. M., \& Romani, R. W. 2002, VizieR Online Data Catalog, 338, 61001

Gotthelf, E. V. 2003, ApJ, 591, 361

Gotthelf, E. V., Halpern, J. P., \& Dodson, R. 2002, ApJ, 567, L125

Hartman, R. C., Bertsch, D. L., Bloom, S. D., Chen, A. W., Deines-Jones, P., Esposito, J. A., Fichtel, C. E., Friedlander, D. P., Hunter, S. D., McDonald, L. M., Sreekumar, P., Thompson, D. J., Jones, B. B., Lin, Y. C., Michelson, P. F., Nolan, P. L., Tompkins, W. F., Kanbach, G., Mayer-Hasselwander, H. A., Mücke, A., Pohl, M., Reimer, O., Kniffen, D. A., Schneid, E. J., von Montigny, C., Mukherjee, R., \& Dingus, B. L. 1999, ApJS, 123, 79

Helfand, D. J., Gotthelf, E. V., \& Halpern, J. P. 2001, ApJ, 556, 380

Kaspi, V. M., Roberts, M. S. E., \& Harding, A. K. 2004, in Compact Stellar X-ray Sources, ed. W. H. G. Lewin \& M. van der Klis (United Kingdom: Cambridge University Press), in press

Kaspi, V. M., Roberts, M. S. E., Vasisht, G., Gotthelf, E. V., Pivovaroff, M., \& Kawai, N. 2001, ApJ, 560, 371

Kifune, T., Tanimori, T., Ogio, S., Tamura, T., Fujii, H., Fujimoto, M., Hara, T., Hayashida, N., Kabe, S., Kakimoto, F., Matsubara, Y., Mizumoto, Y., Muraki, Y. ., Suda, T., Teshima, M., Tsukagoshi, T., Watase, Y., Yoshikoshi, T., Edwards, P. G., Patterson, J. R., Roberts, M. D., Rowell, G. P., \& Thornton, G. J. 1995, ApJ, 438, L91

Kramer, M., Bell, J. F., Manchester, R. N., Lyne, A. G., Camilo, F., Stairs, I. H., D’Amico, N., Kaspi, V. M., Hobbs, G., Morris, D. J., Crawford, F., Possenti, A., Joshi, B. C., McLaughlin, M. A., Lorimer, D. R., \& Faulkner, A. J. 2003, MNRAS, 342, 1299

McLaughlin, M. A. \& Cordes, J. M. 2003, ArXiv Astrophysics e-prints

Mel'nik, A. M. \& Efremov, Y. N. 1995, Sov. Astron. Lett., 21, 10 
Migliazzo, J. M., Gaensler, B. M., Backer, D. C., Stappers, B. W., van der Swaluw, E., \& Strom, R. G. 2002, ApJ, 567, L141

Ng, C.-Y. \& Romani, R. W. 2004, ApJ, 601, 479

Pavlov, G. G., Zavlin, V. E., Sanwal, D., Burwitz, V., \& Garmire, G. P. 2001, ApJ, 552, L129

Possenti, A., Cerutti, R., Colpi, M., \& Mereghetti, S. 2002, A\&A, 387, 993

Rankin, J. M. 1993, ApJ, 405, 285

Rickett, B. J. 1977, ARAA, 15, 479

Roberts, M. S. E. 2003, in ASP Conf. Ser. 302: Radio Pulsars, 337

Roberts, M. S. E., Hessels, J. W. T., Ransom, S. M., Kaspi, V. M., Freire, P. C. C., Crawford, F., \& Lorimer, D. R. 2002, ApJ, 577, L19

Roberts, M. S. E., Romani, R. W., \& Kawai, N. 2001, ApJS, 133, 451

Roberts, M. S. E., Tam, C. R., Kaspi, V. M., Lyutikov, M., Vasisht, G., Pivovaroff, M., Gotthelf, E. V., \& Kawai, N. 2003, ApJ, 588, 992

Romani, R. W. 1996, ApJ, 470, 469

Romani, R. W. \& Yadigaroglu, I.-A. 1995, ApJ, 438, 314

Standish, E. M. 1998, Interoffice Memo. 312.F-98-048. Pasadena: JPL

Thompson, D. J., Bailes, M., Bertsch, D. L., Cordes, J., D’Amico, N., Esposito, J. A., Finley, J., Hartman, R. C., Hermsen, W., Kanbach, G., Kaspi, V. M., Kniffen, D. A., Kuiper, L., Lin, Y. C., Lyne, A., Manchester, R., Matz, S. M., Mayer-Hasselwander, H. A., Michelson, P. F., Nolan, P. L., Ögelman, H., Pohl, M., Ramanamurthy, P. V., Sreekumar, P., Reimer, O., Taylor, J. H., \& Ulmer, M. 1999, ApJ, 516, 297

Weisskopf, M. C., Hester, J. J., Tennant, A. F., Elsner, R. F., Schulz, N. S., Marshall, H. L., Karovska, M., Nichols, J. S., Swartz, D. A., Kolodziejczak, J. J., \& O’Dell, S. L. 2000, ApJ, 536, L81

Yadigaroglu, I.-A. \& Romani, R. W. 1997, ApJ, 476, 347

Zhang, B. \& Harding, A. K. 2000, ApJ, 532, 1150 
Table 1. Measured and Derived Parameters for PSR J2021+3651

\begin{tabular}{|c|c|}
\hline Parameter & Value \\
\hline Right ascension $(\mathrm{J} 2000.0)^{\mathrm{a}}$ & $20^{h} 21^{m} 05^{s} .46(5)$ \\
\hline Declination $(\mathrm{J} 2000.0)^{\mathrm{a}}$ & $+36^{\circ} 51^{\prime} 04^{\prime \prime} .8(7)$ \\
\hline Galactic longitude $l(\mathrm{deg})^{\mathrm{a}}$ & 75.23 \\
\hline Galactic latitude $b(\mathrm{deg})^{\mathrm{a}}$ & +0.11 \\
\hline $\mathrm{DM}\left(\mathrm{pc} \mathrm{cm}{ }^{-3}\right)$ & $369.2(2)$ \\
\hline \multicolumn{2}{|l|}{ Preglitch } \\
\hline Pulse frequency $\nu\left(\mathrm{s}^{-1}\right)$ & $9.64113518458(16)$ \\
\hline Frequency derivative $\dot{\nu}\left(\mathrm{s}^{-2}\right)$ & $-8.886500(24) \times 10^{-12}$ \\
\hline Epoch (MJD) & 52407.389 \\
\hline Range of MJDs & $52305-52616$ \\
\hline RMS Residuals (ms) & 3.0 \\
\hline \multicolumn{2}{|l|}{ Postglitch } \\
\hline Pulse frequency $\nu\left(\mathrm{s}^{-1}\right)$ & $9.64091197572(12)$ \\
\hline Frequency derivative $\dot{\nu}\left(\mathrm{s}^{-2}\right)$ & $-8.932592(12) \times 10^{-12}$ \\
\hline Epoch (MJD) & 52730 \\
\hline Range of MJDs & $52645-53009$ \\
\hline RMS Residuals (ms) & 9.3 \\
\hline \multicolumn{2}{|c|}{ Glitch Parameters } \\
\hline Epoch (MJD) & $52629.97-52630.16$ \\
\hline Frequency jump $\Delta \nu / \nu$ & $2.587(2) \times 10^{-6}$ \\
\hline Frequency derivative jump $\Delta \dot{\nu} / \dot{\nu}$ & $6.2(3) \times 10^{-3}$ \\
\hline \multicolumn{2}{|c|}{ Derived Parameters } \\
\hline Spin-down Luminosity $\dot{E}^{\mathrm{b}}\left(\operatorname{ergs~s}^{-1}\right)$ & $3.4 \times 10^{36}$ \\
\hline Surface Magnetic Field $B^{\mathrm{c}}(\mathrm{G})$ & $3.2 \times 10^{12}$ \\
\hline Characteristic Age $\tau_{c} \equiv P / 2 \dot{P}(\mathrm{kyr})$ & 17 \\
\hline
\end{tabular}

Note. - Figures in parentheses represent uncertainty in the least-significant digits quoted equal to 3 times errors given by TEMPO. 


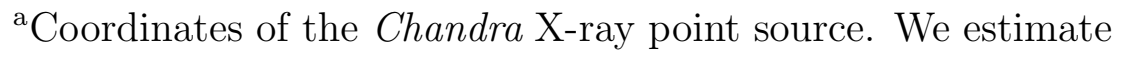
a positional error of $\sim 1^{\prime \prime}$ by comparing the position of another bright X-ray point source on the $\mathrm{S} 3$ chip with a catalogued optical counterpart.

$$
{ }^{\mathrm{b}} \dot{E} \equiv 4 \pi^{2} I \dot{P} / P^{3} \text { with } I=10^{45} \mathrm{~g} \mathrm{~cm}^{2} .
$$

${ }^{\mathrm{c}}$ Assuming standard magnetic dipole spindown: $B \equiv 3.2 \times$ $10^{19}(P \dot{P})^{1 / 2}$ Gauss. 
Table 2. Torus Fit Parameters

\begin{tabular}{ll}
\hline \hline Parameter $^{\mathrm{a}}$ & \multicolumn{1}{c}{ Value } \\
\hline$\Psi$ & $47^{\circ} \pm 1^{\circ}$ \\
$\zeta$ & $83^{\circ} \pm 1^{\circ}$ \\
$r$ & $8.6^{\prime \prime} \pm 0.3^{\prime \prime}$ \\
$\delta^{\mathrm{b}}$ & $1.2^{\prime \prime}$ \\
$\beta_{T}{ }^{\mathrm{c}}$ & $\sim 0.8$ \\
$d$ & $3.7^{\prime \prime} \pm 0.2^{\prime \prime}$ \\
\hline
\end{tabular}

${ }^{a}$ Explanation of parameters in $\S 4.4$

${ }^{\mathrm{b}}$ Held fixed in the global fit.

cThe Doppler boosting is likely being dominated by the bright clump to the south-west of the pulsar. 


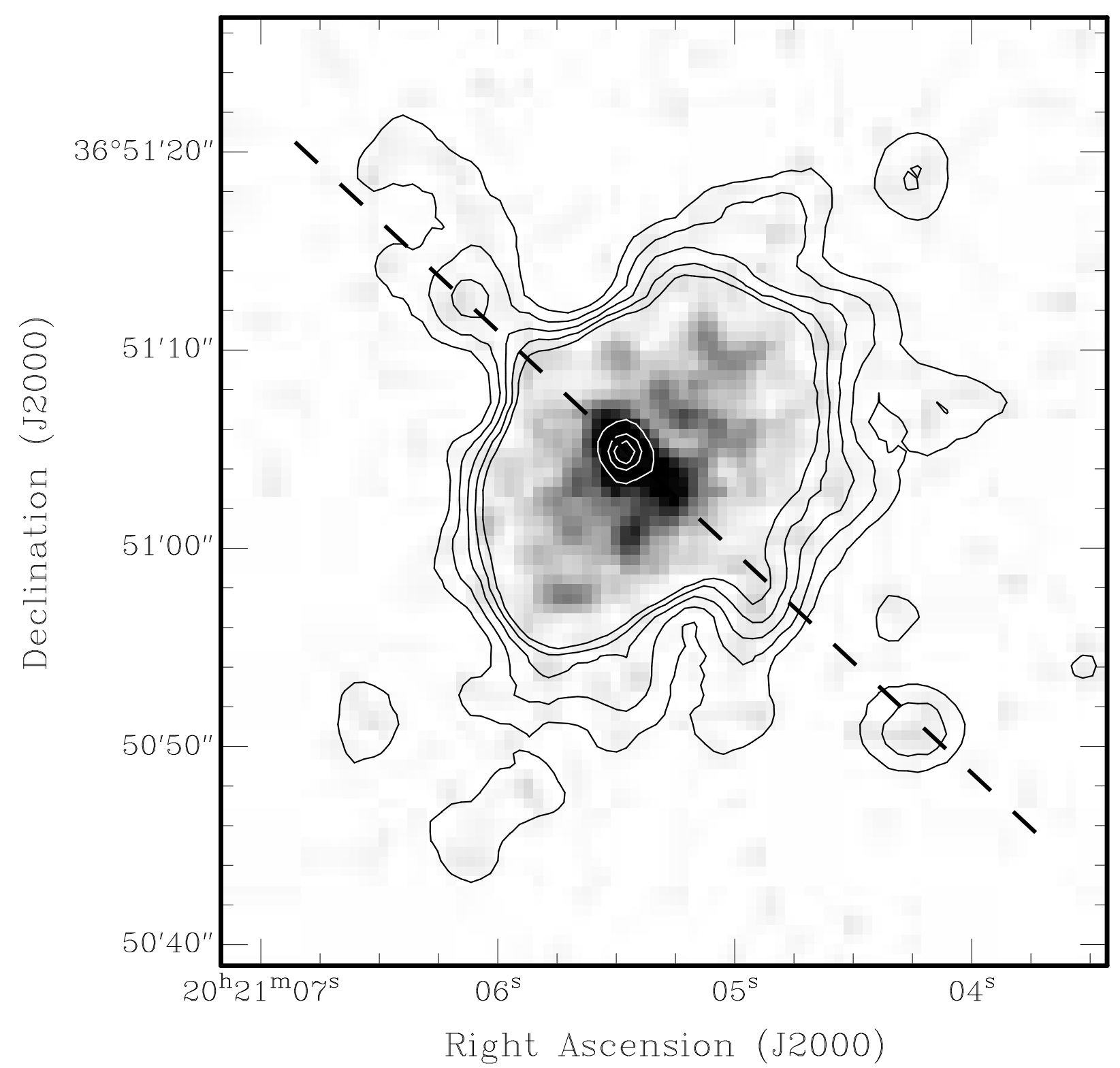

Fig. 1.- A 19.0-ks Chandra ACIS-S image (0.3-7.0 keV) of PSR J2021+3651 and its inner nebula. The greyscale shows the image smoothed with a 1.2" (FWHM) gaussian and scaled to bring out fine structure in the nebula. The black contours are the same image overlaid, but smoothed with a $3.5^{\prime \prime}$ gaussian and scaled to bring out faint extended emission including what may possibly be faint jets along the axis of the nebula. The white contours indicate the point source and are shown to distinguish it from the bright bar running along the axis of the nebula. The dashed line shows the best-fit position angle $\Psi=47^{\circ} \pm 1^{\circ}$ of the torus fit (see $\$ 4.4$ ), which in analogy with the Crab pulsar and nebula likely corresponds to the projected spin axis of the pulsar. 

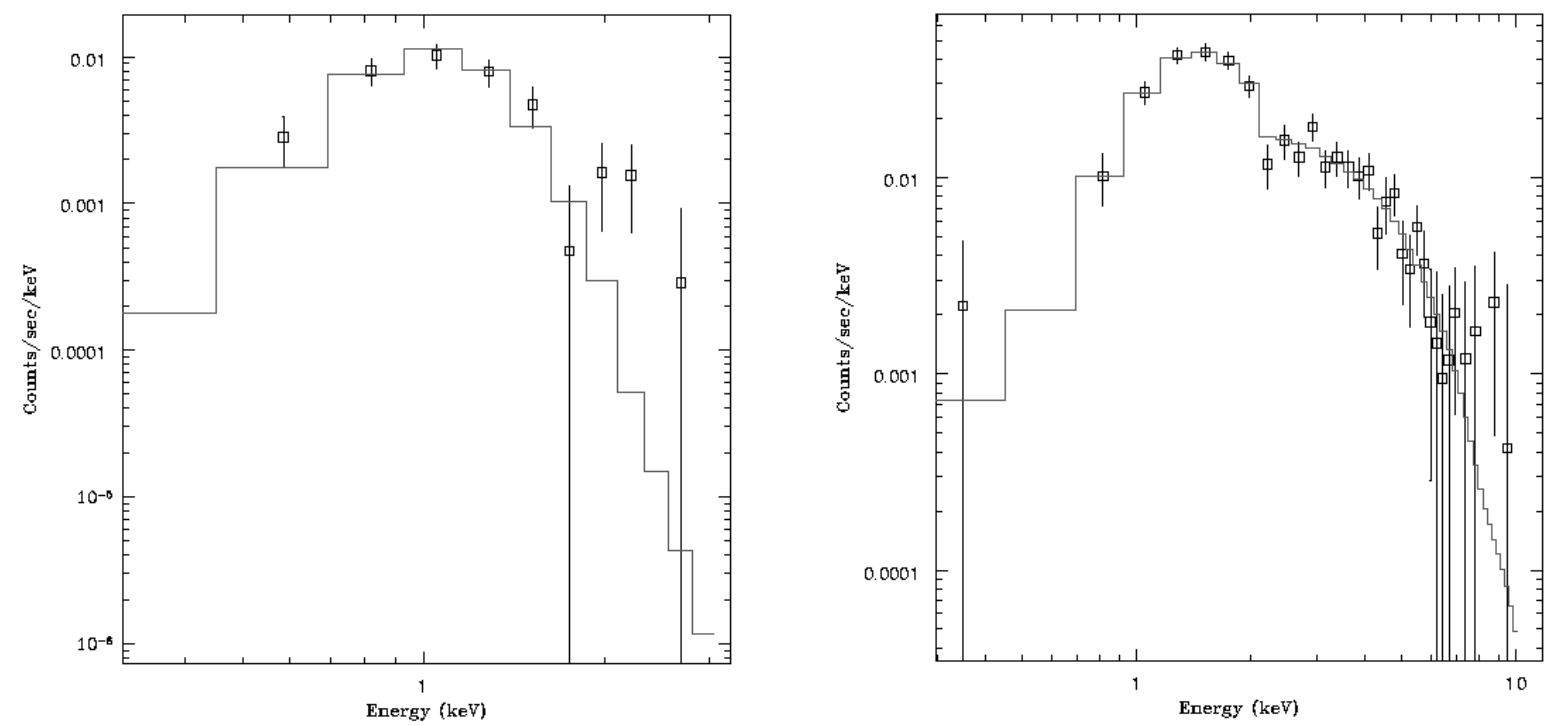

Fig. 2.- Left: The background-subtracted spectrum of the point source, fit using an absorbed black-body model in Sherpa (CIAO 3.01). The best-fit temperature and $90 \%$ confidence interval is $k T_{\infty}=0.15 \pm 0.02 \mathrm{keV}$ with a $0.3-10.0 \mathrm{keV}$ absorbed flux of $(2.8 \pm 0.2) \times 10^{-14}$ erg $\mathrm{cm}^{-2} \mathrm{~s}^{-1}$. The $\mathrm{n}_{\mathrm{H}}$ was fixed at $7.8 \times 10^{21} \mathrm{~cm}^{-2}$, that found by fitting the nebular spectrum. Right: The background-subtracted spectrum of the nebula fit with an absorbed power-law model. The best-fit parameters and $90 \%$ confidence intervals are $\mathrm{n}_{\mathrm{H}}=\left(7.8_{-1.4}^{+1.7}\right) \times 10^{21} \mathrm{~cm}^{-2}$, $\Gamma=1.7_{-0.2}^{+0.3}$, and an absorbed flux of $(1.2 \pm 0.1) \times 10^{-12} \mathrm{erg} \mathrm{cm}^{-2} \mathrm{~s}^{-1}(0.3-10.0 \mathrm{keV})$. 


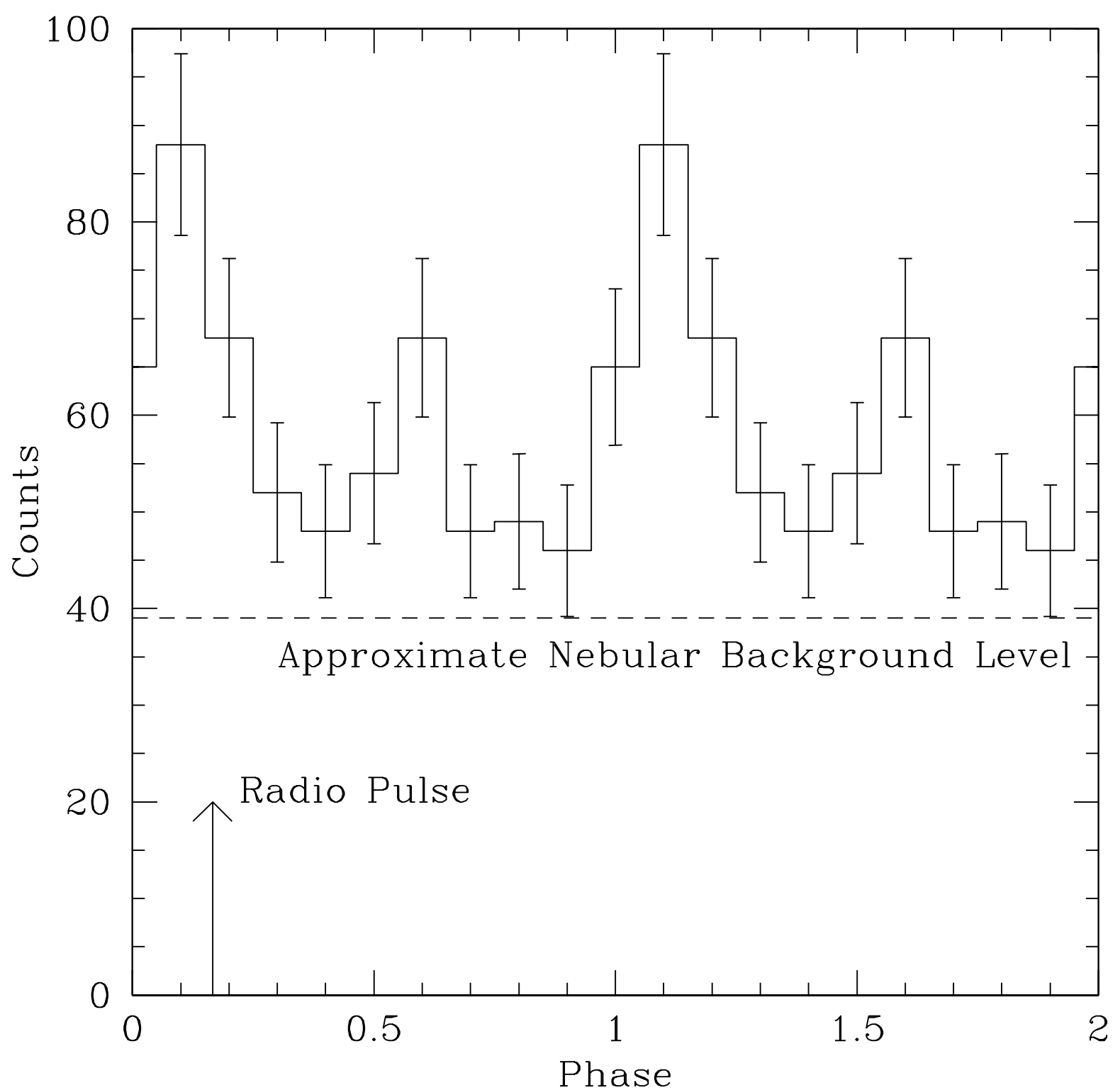

Fig. 3.- Chandra continuous clocking data of PSR J2021+3651 folded using a local radio ephemeris for the pulsar. Two cycles are plotted for clarity and there are ten bins across the profile. The phase of the radio pulse is indicated by an arrow. The dashed line indicates the approximate background level. Based on this, there are $\sim 69$ pulsed counts and we estimate that $\sim 190$ counts are from the pulsar. This gives a pulsed fraction of $\sim 37 \%$. 


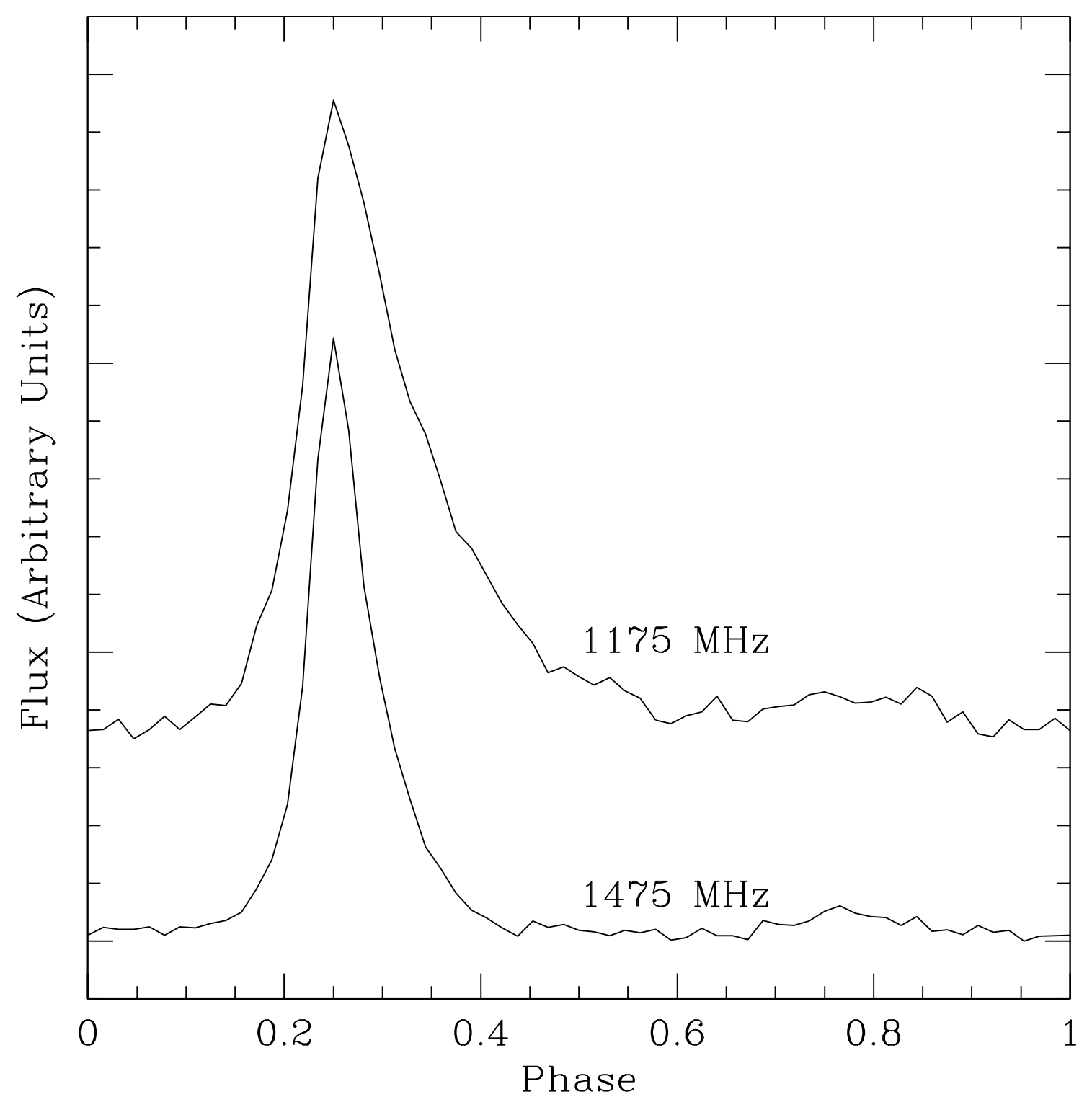

Fig. 4.- Summed pulse profiles of PSR J2021+3651 at center frequencies of $1175 \mathrm{MHz}$ (top) and $1475 \mathrm{MHz}$ (bottom). The peak fluxes of the two profiles are normalized and the pulse phases aligned at the pulse peak. By fitting the profiles to a gaussian convolved with an exponential we calculate a scattering time $\tau_{s c}=17.7 \pm 0.9 \mathrm{~ms}$ at $1 \mathrm{GHz}$. 

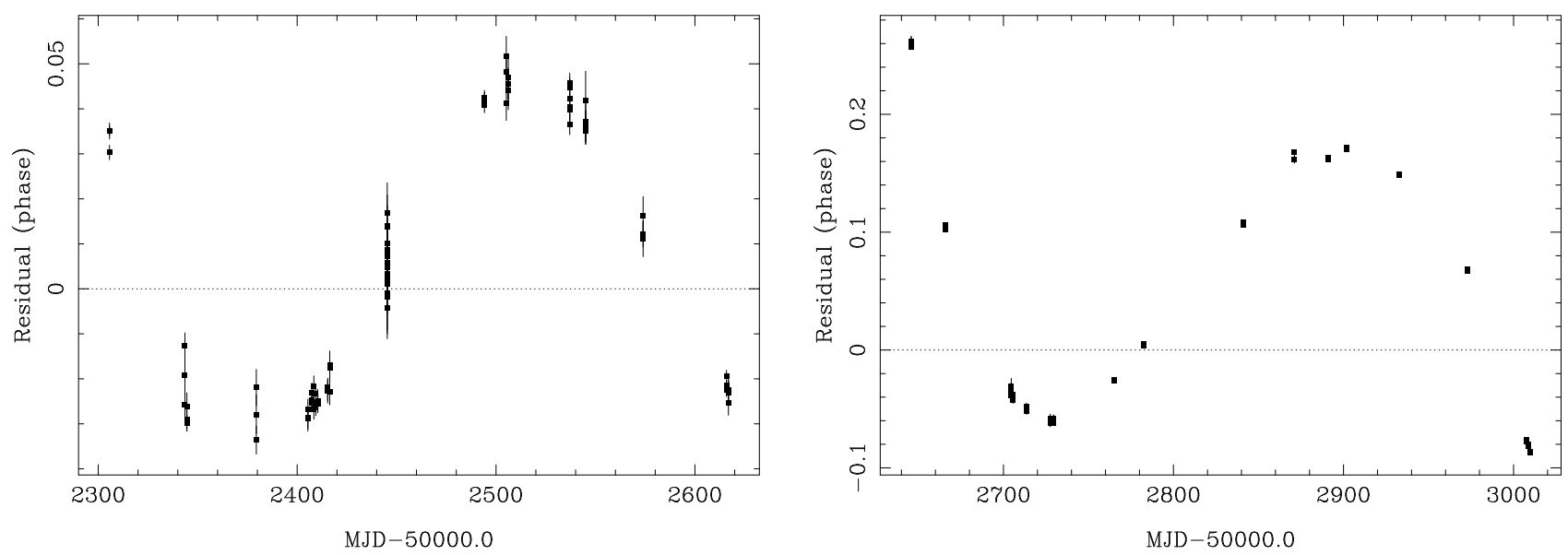

Fig. 5.- Residuals from phase-coherent timing of PSR J2021+3651 fitting only for period and the first period derivative. The residuals can be rendered featureless by fitting for a period second derivative. Alternately, the residuals can be rendered featureless by fitting for position (instead of keeping it fixed at the X-ray position). However, the fitted positions from the pre- and post-fit ephemerides are inconsistent suggesting that timing noise dominates the residuals, rather than an incorrect position. Left: pre-glitch residuals. Right: post-glitch residuals.

Fig. 6. - Left: $32^{\prime \prime} \times 32^{\prime \prime}$ cutout of the central PWN. Middle: best-fit double torus plus point source model. Right: best-fit single torus plus point source model. 